DOI: https://doi.org/10.46296/ig.v3i5.0012

\title{
DESARROLLO E IMPLEMENTACIÓN DE APLICACIÓN MÓVIL PARA LA DIFUSIÓN DE PUBLICACIONES DE LA EDITORIAL ULEAM
}

\section{DEVELOPMENT AND IMPLEMENTATION OF MOBILE APPLICATION FOR THE DISSEMINATION OF PUBLICATIONS OF THE EDITORIAL ULEAM}

\author{
Zambrano-Pilay Enrique ${ }^{1 *}$; Vélez-Vélez Luis²; Almeida-Zambrano Edison³ \\ ${ }^{1}$ Universidad Laica Eloy Alfaro de Manabí, ULEAM. Manta, Ecuador. \\ ${ }^{2}$ Universidad Laica Eloy Alfaro de Manabí, ULEAM. Manta, Ecuador. \\ ${ }^{3}$ Profesor de la Universidad Laica Eloy Alfaro de Manabí, ULEAM. Manta, Ecuador.
}

*Correo: e1315391167@live.uleam.edu.ec

\begin{abstract}
Resumen
La publicación y divulgación científica son parámetros que reflejan la calidad de la educación superior, más aún si se fundamentan en herramientas tecnológicas. Es por esta razón que desde la Editorial Universitaria Uleam nace la necesidad de implementar una plataforma móvil que brinde a la comunidad nacional e internacional, contenido científico y conocimientos ancestrales de acceso abierto. El presente proyecto de investigación y desarrollo tecnológico está basado en la creación e implementación de un aplicativo móvil que permitirá visualizar los libros y artículos científicos publicados por la Editorial Universitaria y a su vez difundir entre la comunidad con escaso acceso debido a limitaciones asociadas con la dificultad visual. Esta aplicación móvil se diseñará bajo una plataforma Android y se enlazará con la base de datos de la plataforma institucional SEGUP, en la que se encuentran alojados los textos científicos publicados.
\end{abstract}

Palabras clave: publicación, aplicación móvil, libros, artículos.

\begin{abstract}
Scientific publication and dissemination are parameters that reflect the quality of higher education, even more so if they are based on technological tools. It is for this reason that from the University Publishing Uleam the need arises to implement a mobile platform that provides the national and international community with scientific content and ancient knowledge of open access. This technological research and development project are based on the creation and implementation of a mobile application that will allow viewing the scientific books and articles published by the University Editorial and, in turn, disseminating it to the community with little access due to limitations associated with visual difficulties. This mobile application will be designed under an Android platform and will be linked to the database of the SEGUP institutional platform, where the published scientific texts are housed.
\end{abstract}

Keywords: publication, mobile application, books, articles.

Información del manuscrito:

Fecha de recepción: 16 de noviembre de 2019

Fecha de aceptación: 20 de diciembre de 2019

Fecha de publicación: 10 de enero de 2020 


\section{Introducción}

Moon+ Reader es un lector de libros alojado en Google Play Store, con potentes controles y completas funciones que le permiten la lectura de miles de libros electrónicos de forma gratuita; es compatible con las bibliotecas en línea de libros electrónicos. Además, permite la lectura de libros locales y soporta diversos formatos de ebook como PDF, DOCX, TXT, HTML, RAR, ZIP, incluido Epub3. Por otra parte, esta aplicación móvil cuenta con una versión pro o de paga, la cual aporta diferentes beneficios para los usuarios (Menéndez, 2015).

El aplicativo móvil "eBoox" está alojado en Google Play Store, siendo este un lector de libros electrónicos en formato: fb2, ePub, doc, docx, mobi, prc, txt, rtf, odt, html y archivos zip, siendo más agradable para Android. Además, permite cargar los documentos fácilmente desde cualquier carpeta del móvil y tarjeta SD, así como desde la nube y diversos navegadores web. En la actualidad cuenta con cuatro libros cargados en la aplicación para las respectivas pruebas de funcionalidad por parte de los usuarios (Pubgen, 2019).
El presente trabajo muestra la competitividad e innovación de los sistemas relacionados al desarrollo e implementación de una aplicación móvil enlazada con la base de datos de la plataforma SEGUP, que permitirá difundir los libros y revistas científicas publicadas en la Editorial Uleam, además de tener un enfoque inclusivo al incorporar lectura asistida por voz, ayudando a personas con discapacidad visual o problemas de lectura.

Por lo mencionado anteriormente, en este trabajo se pretende dar a conocer una mejor comprensión de la implementación, funcionamiento, y el impacto que puede tener el desarrollo de la plataforma SEGUP, así como el lenguaje de programación y la metodología.

\section{Metodología}

\subsection{Diseño metodológico}

Se presenta la ejecución de la metodología de trabajo SCRUM para la gestión y desarrollo de la aplicación móvil creada con fines divulgativos de las publicaciones científicas de la Editorial Uleam, Universidad Laica Eloy Alfaro de Manabí, ubicada en Manta, Ecuador. Esta aplicación fue diseñada con 
enfoque inclusivo para personas con discapacidad visual y con problemas de lectura, además se agrupan conocimientos multidisciplinarios y saberes ancestrales. Los procesos y mecanismos que la Editorial requirió para la implementación y desarrollo de la aplicación móvil, se especifican a continuación:

- Registro: en este módulo los usuarios pueden registrarse en la aplicación con sus datos personales básicos (nombres, apellidos, cédula o pasaporte, nombre de usuario, e-mail, género y contraseña).

- Login: en este módulo los usuarios pueden ingresar al sistema con su usuario y contraseña correspondientes al registro.

- Gestión y revisión de libros y de artículos científicos: permite la revisión y lectura de los libros publicados en la del conocimiento y la colección multidisciplinaria Pachamama. Además, cuenta con la lectura asistida por interprete de voz, dando el enfoque inclusivo a personas con discapacidad auditiva o con problemas de lectura.

\subsection{Especificaciones técnicas}

En el desarrollo e implementación del presente trabajo se utilizaron las siguientes herramientas informáticas:

- Lenguaje de programación.

- Servidor.

- Frameworks para front-end.

- Frameworks para back-end.

- Base de datos.

- Entorno de desarrollo integrado.

\subsection{Componentes}

\subsubsection{Inclusividad}

La inclusividad es un enfoque que responde positivamente a la diversidad de las personas y a las diferencias individuales, entendiendo que la diversidad no es un problema, sino una oportunidad para el enriquecimiento de la sociedad, a través de la activa participación en la vida familiar, en la educación, en el trabajo y en general en todos los procesos sociales, culturales y en las comunidades. El principal pilar de la inclusión es el reconocimiento de que todas las personas tienen habilidades $y$ potencialidades propias, distintas a las de los demás, por lo que las distintas necesidades exigen 
respuestas diversas o diferentes. (Travieso \& Ribera, 2008).

\subsubsection{Smartphone}

Un smartphone (teléfono inteligente en español) es un dispositivo electrónico que funciona como un teléfono móvil con características similares a las de un ordenador personal. Es un elemento a medio camino entre un teléfono móvil clásico y una PDA ya que permite hacer llamadas y enviar mensajes de texto como un móvil convencional pero además incluye características cercanas a las de un ordenador personal. Una característica importante de casi todos los teléfonos inteligentes es que permiten la instalación de programas para incrementar el procesamiento de datos y la conectividad. Estas aplicaciones pueden ser desarrolladas por el fabricante del dispositivo, por el operador o por un tercero (Alonso et al., 2011).

\subsubsection{Aplicación móvil}

Se denomina aplicación móvil o app a toda aplicación informática diseñada para ser ejecutada en teléfonos inteligentes, tabletas y otros dispositivos móviles. Por lo general se encuentran disponibles a través de plataformas de distribución, operadas por las compañías propietarias de los sistemas operativos móviles como Android, iOS, BlackBerry OS y Windows Phone, entre otros. Con el explosivo desarrollo de los dispositivos móviles en los últimos años, el término app se volvió popular rápidamente, a tal punto que en 2010 fue incluido por la American Dialect Society como Word of the Year (Palabra del Año) (Santiago \& Trabaldo, 2015).

\subsubsection{Android}

Según Alonso et al. (2011) nos dice que Android es un sistema operativo móvil basado en Linux y Java que ha sido liberado bajo la licencia Apache versión 2. El sistema busca, nuevamente, un modelo estandarizado de programación que simplifique las labores de creación de aplicaciones móviles y normalice las herramientas en el campo de la telefonía móvil. Lo que se busca es que los programadores sólo tengan que desarrollar sus creaciones una única vez y así ésta sea compatible con diferentes terminales. 


\subsubsection{Servicio Web}

El servicio Web, se podría definir como un amplio sistema multimedia de acceso a información heterogénea distribuida por toda la red en forma de documentos hipertextuales (hipertextos) (Cobo, 2005)

Según Cobo (2005) el término hipertexto que empezó a hacerse popular a partir de la aparición de este servicio tiene, sin embargo, su definición en un trabajo de Ted Nelson en 1965. La definición original del término es:

"Un cuerpo de material escrito o gráfico interconectado de un modo complejo que no se puede representar convenientemente sobre el papel; puede contener anotaciones, adiciones y notas de los estudiosos que lo examinan".

No obstante, Cobo (2005) da una definición más moderna y aplicable al concepto de hipertexto en Internet, se podría decir que un hipertexto es un documento multimedia, es decir, integrado bajo una plataforma informática con todas las tecnologías de la información, y que incorpora relaciones estructurales que enlazan el documento con otros documentos o recursos.

\subsubsection{HTML}

HTML es un lenguaje artificial que los ordenadores son capaces de interpretar, se encuentra diseñado para que los programadores redacten instrucciones que los navegadores ejecutan normalmente para originar la página web. Es decir, HTML es un lenguaje de programación o un idioma que la maquina entiende para procesar una respuesta (Equipo Vértice, 2009; Humberto, 2018).

\subsubsection{Android Studio}

Cañarte et al. (2018) señalan que Android Studio es el entorno de desarrollo integrado oficial (IDE) para la creación de apps basadas en los sistemas de Android, basado en IntelliJ IDEA. Además del potente editor de códigos y las herramientas para desarrolladores de IntelliJ, Android Studio ofrece aún más funciones que aumentan la productividad el usuario cuando desarrolla apps para Android, como las siguientes:

- Un sistema de compilación flexible basado en Gradle. 
- Un emulador rápido y cargado de funciones.

- Un entorno unificado donde puedes desarrollar para todos los dispositivos Android.

- Ejecución al instante para aplicar cambios a tu app en funcionamiento sin necesidad de compilar un APK nuevo.

- Integración con GitHub y plantillas de código para ayudarte a compilar funciones de aplicaciones comunes y también importar código de ejemplo.

- Variedad de marcos de trabajo y herramientas de prueba.

- Herramientas de Lint para identificar problemas de rendimiento, usabilidad y compatibilidad de la versión, entre otros.

- Compatibilidad con $\mathrm{C}++$ y NDK.

\subsubsection{JavaScript}

Eguíluz (2012) indica que JavaScript es un lenguaje de programación que se utiliza principalmente para crear páginas web dinámicas, es decir que incorporan efectos como textos que aparecen y desaparecen, animaciones, acciones que se activan al pulsar botones y ventanas con mensajes de aviso al usuario. Técnicamente, JavaScript es un lenguaje de programación interpretado, por lo que no es necesario compilar los programas para ejecutarlos. En otras palabras, los programas escritos con JavaScript se pueden probar directamente en cualquier navegador sin necesidad de procesos intermedios. Sin embargo, López \& Quijije (2018) señalan que se trata de un lenguaje script compacto, basado en objetos y guiados por eventos diseñados específicamente para el desarrollo de aplicaciones cliente-servidor dentro del internet.

\subsubsection{PHP}

Según Cobo (2005) PHP es un lenguaje interpretado del lado del servidor que surge dentro de la corriente denominada código abierto (open source). Se caracteriza por su potencia, versatilidad, robustez y modularidad. Al igual que ocurre con tecnologías similares, los programas son integrados directamente dentro del código HTML. Además, este es uno de los lenguajes más utilizados actualmente en el desarrollo de aplicaciones web y viene experimentado un constante crecimiento en su nivel de utilización en Internet. 


\subsubsection{MySQL}

MySQL es un sistema de gestión de bases de datos relacional $y$ desarrollado bajo licencia dual GPL/Licencia comercial por Oracle Corporation. Se considerada como la base datos open source más popular del mundo, y una de las más populares en general junto a Oracle y Microsoft SQL Server, sobre todo para entornos de desarrollo web (Lazo, 2018).

\subsubsection{Sprint}

Es una iteración o ciclo repetitivo de trabajo semejante, que produce un incremente de producto o sistema. Los períodos iterativos son menores a un mes, y usualmente mayores a una semana. La duración es fija a lo largo de todo el trabajo, y todos los equipos que trabajan en el mismo sistema o producto usan la misma duración de ciclo (Deemer et al., 2012).

\subsubsection{Google Play Store}

Es una plataforma de distribución digital de aplicaciones móviles para los dispositivos con sistema operativo Android, así como una tienda en línea desarrollada y operada por Google. Esta plataforma permite a los usuarios navegar y descargar

aplicaciones

desarrolladas mediante Android SDK, juegos, música, libros, revistas y películas (Difabio et al., 2016).

\subsubsection{SCRUM}

Chávez-Quiroz \& Valdez-Menéndez (2018) concuerdan

con SCRUMstudyTM (2016) señalando que Scrum es una de las metodologías ágiles más populares. Es adaptable, iterativa, rápida, flexible y eficaz, diseñada para ofrecer un valor significativo de forma rápida en todo el proyecto. Scrum garantiza transparencia en la comunicación y crea un ambiente de responsabilidad colectiva y de progreso continuo. Una fortaleza clave de Scrum radica en el uso de equipos interfuncionales, autoorganizados, y empoderados que dividen su trabajo en ciclos de trabajos cortos y concentrados llamados Sprints.

\section{Resultados y discusión}

La aplicación de tres herramientas de recolección de información permitió lograr una mejor compresión del problema y así plantear una propuesta acorde a las necesidades encontradas en la Editorial Uleam, esta información fue 
de vital importancia y da paso a la elicitación de requisitos para realizar el proceso de la difusión de libros y revistas científicas, así mismo se constató que las obras publicadas carecen de mecanismos para poder ser leídas por personas con discapacidad visual o con problemas de lectura, tomando como punto de partida estos factores para darle un giro inclusivo al proyecto.

La plataforma que se adecua más a la presente propuesta es la móvil, con tecnología Android, ya que la Editorial carece de una aplicación para difundir sus trabajos publicados, así mismo la plataforma estará dirigida a la comunidad en general, puesto que la mayoría de personas posee dispositivos móviles con sistema operativo Android.

La plataforma informática que implementará la Editorial Uleam tiene una gran acogida en la comunidad universitaria, ya que permite difundir los trabajos publicados. Además, el proyecto tiene enfoque inclusivo, puesto que las personas con discapacidad visual o con problemas de lectura también pueden revisar las obras que se alojen en la aplicación.

\subsection{Arquitectura}

La arquitectura del software es el diseño global de la propuesta y permite obtener el funcionamiento al más alto nivel de la estructura de un sistema. En otras palabras, tiene que ver con el diseño e implementación de estructuras de software de alto nivel. Es el resultado de ensamblar los elementos informáticos utilizados en el proyecto de forma que permitan satisfacer la mayor funcionalidad y requerimientos de desempeño de un sistema, así como requerimientos no funcionales, como la confiabilidad. La figura 1 muestra la arquitectura total de la aplicación móvil "InLector".

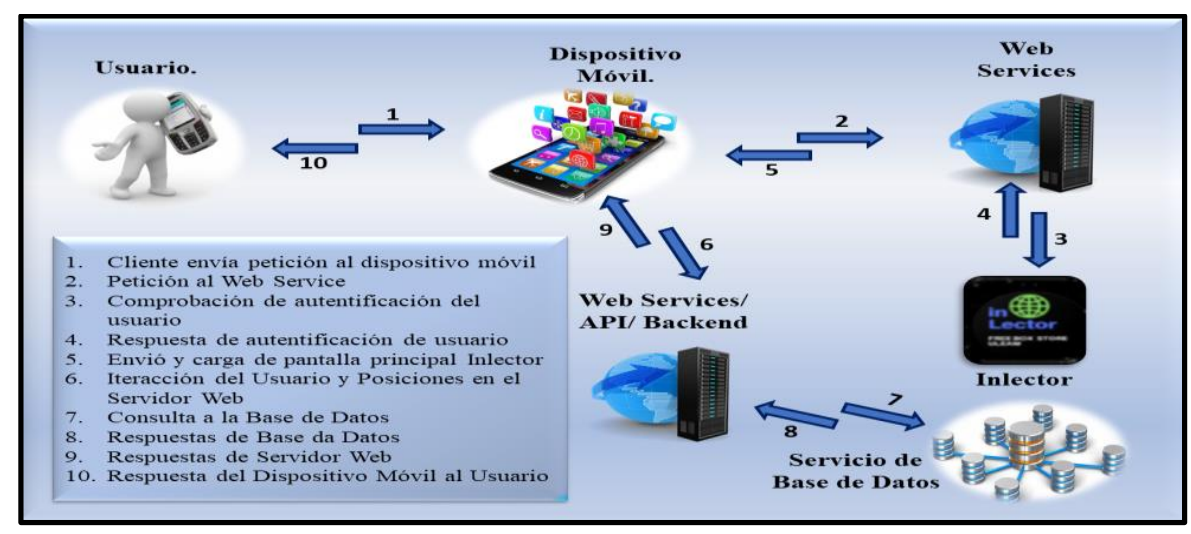

Figura 1. Arquitectura total de la aplicación móvil "InLector" 


\subsubsection{Modelo de base de datos}

Un modelo de base de datos muestra la estructura lógica de la base, incluidas las relaciones $y$ limitaciones que determinan come se almacenan los datos y come se accede a ellos. En la siguiente figura se muestra el diagrama completo de la base de datos con todas las relaciones y atributos existentes empleados en el proyecto.

\begin{tabular}{|c|c|c|c|c|}
\hline 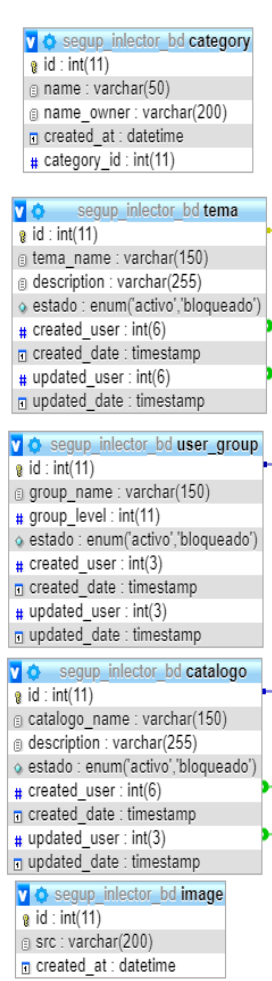 & 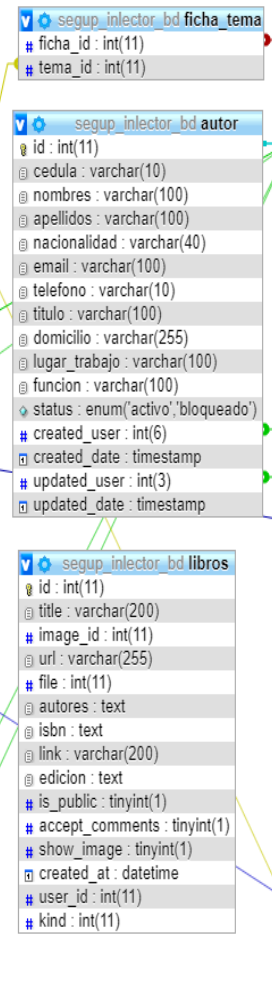 & 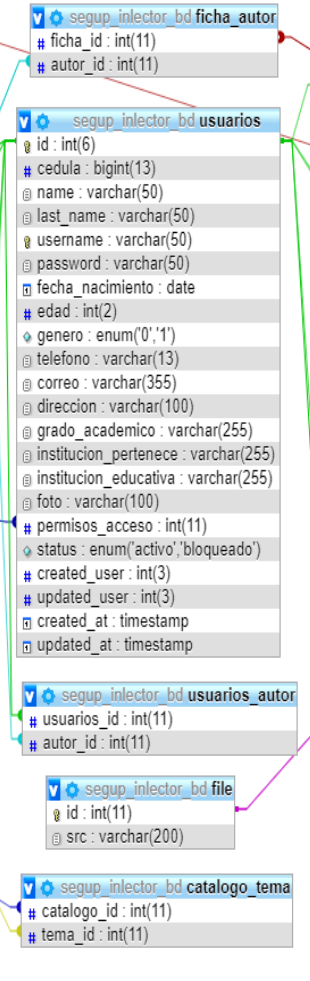 & 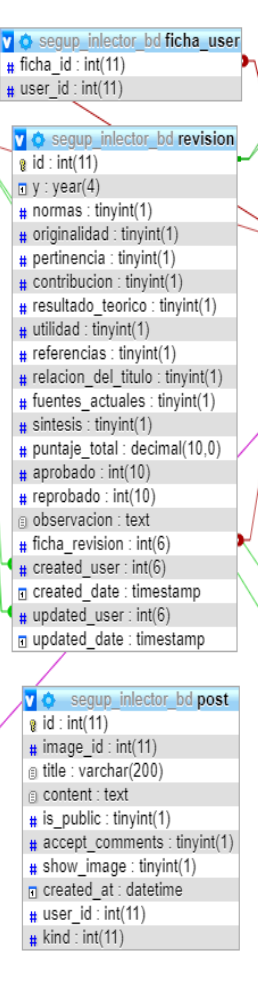 & 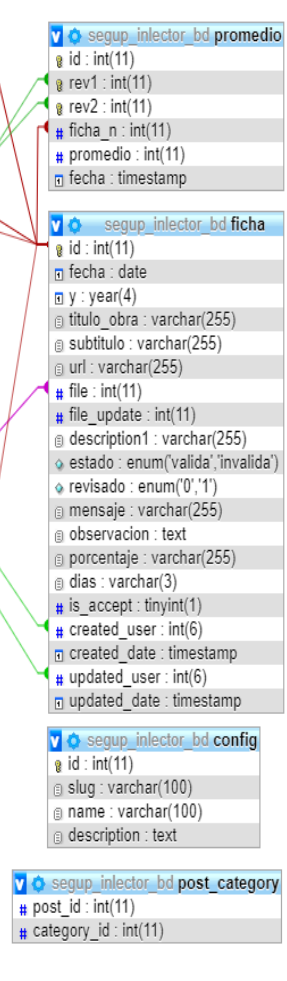 \\
\hline
\end{tabular}

Figura 2. Base de datos de los sistemas SEGUP e InLector

\subsubsection{Implementación de la}

\section{plataforma}

La utilización de la metodología SCRUM durante el desarrollo del trabajo permitió la construcción e implementación de la aplicación móvil con enfoque inclusivo para la difusión científica de publicaciones de la Editorial Uleam. Cabe resaltar que está diseñada para el uso general de la comunidad que desee revisar el contenido de las publicaciones que produce la Editorial.

La implementación de la plataforma fundamenta lo siguiente:

- Sistema modular: las características principales de la aplicación móvil con enfoque inclusivo para la difusión 
científica de publicaciones de la Editorial Uleam permiten desarrollar diferentes fases del proceso de programación, sobre las cuales se realizaron los incrementos de las funcionalidades y modificaciones en el comportamiento de la aplicación, obteniendo en cada incremento un producto con nuevas funciones, apariencias y mejoras.

- Entregas frecuentes y continuas de las funcionalidades implementadas, de forma que se dispongan nuevas versiones de la aplicación móvil en cortos plazos de tiempo y realizando mejoras continuas en el producto.

- Predecible en lo que respecta a la inestabilidad de requisitos en la incorporación de mayores funcionalidades. En este sentido, pueden ocurrir alteraciones en el orden de módulos 0 requerimientos de usuario.

En la figura 3 se presenta un diagrama que especifica la comunicación que tiene el usuario y el sistema mediante las diferentes iteraciones, desde que el usuario se registra hasta el proceso de la visualización de libros y lectura asistida.

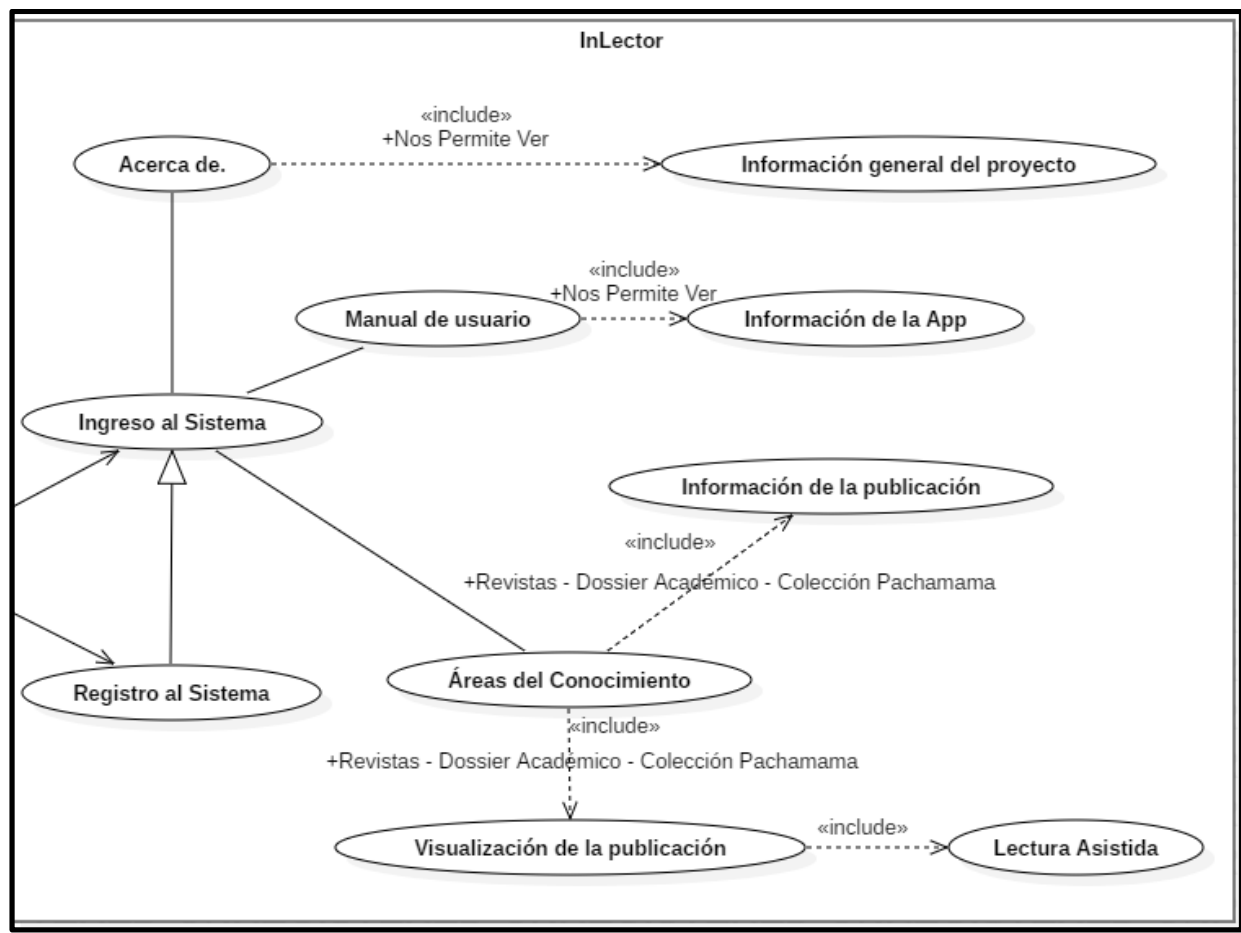

Figura 3. Diagrama de caso de uso general de aplicación móvil InLector 


\section{Conclusiones}

La aplicación móvil se diseñó en la plataforma Android con herramientas de desarrollo como Android Studio, con web servicies para hacer uso y conexión de la base de datos del SEGUP, y con Frameworks adaptados para el desarrollo móvil. Además, se trabajó bajo la metodología Scrum haciendo énfasis a los sprints.

Se diseñó la arquitectura, diagrama de base de datos, y además se definieron los procesos de la metodología Scrum, dividiéndola en tres sprints que devolvían incrementos, revisados y aprobados por los miembros del equipo de trabajo, permitiendo que el desarrollo fuera ágil e interactivo.

Los diferentes requerimientos de información obtenidos de la Editorial Uleam permitieron el diseño, desarrollo e implantación de la aplicación móvil, dando efectos positivos ante la comunidad universitaria, teniendo resultados con impacto científico y muy buena acogida ante la sociedad, ya que permite difundir los trabajos publicados y paralelamente, darle relevancia desde el enfoque inclusivo y participativo.

\section{Bibliografía}

Alonso, A. B., Artime, I. F., Rodríguez, M. Á., \& Baniello, R. G. (2011). Dispositivos móviles. EPSIG Ing. Telecomunicación, Universidad de Oviedo, España.

Cañarte-Montalván, E. R., Tumbaco, C., \& Doralisa, A. (2018). Aplicación móvil para la ubicación y reserva de canchas múltiples en la ciudad de Manta (Doctoral dissertation).

Chávez-Quiroz, G. G., \& Valdez Menéndez, B. S. (2018). Desarrollo e implementación de una plataforma informática para la gestión de servicios automotriz. caso de aplicación: Empresa Xpress (Doctoral dissertation).

Cobo, Á. (2005). PHP y MySQL: Tecnología para el desarrollo de aplicaciones web. Ediciones Díaz de Santos.

Deemer, P., Benefield, G., Larman, C., \& Vodde, B. (2012). Una introducción básica a la teoría y práctica de Scrum. InfoQueue. Enterprise Software Development Series.

Difabio, L. A., Vivas, H. L., \& Muñoz Abbate, H. (2016). Internet de las cosas aplicada a la trazabilidad de la recolección de residuos en ciudades inteligentes. In X Simposio de 
Informática en el Estado (SIE 2016)-JAllO 45 (03 de Febrero, 2016).

Eguíluz-Pérez, J. (2012). Introducción a JavaScript.

Equipo Vértice. (2009). Diseño básico de páginas web en HTML. Editorial Vértice.

Humberto, R. F. M. (2018). Desarrollo e implementación de un sistema web para mejorar la administración de los procesos internos y el servicio al cliente de la pyme gráficas Rivas, implementando también una herramienta de inteligencia artificial chatbot (Doctoral dissertation, Universidad de Guayaquil. Facultad de Ciencias Matemáticas y Físicas. Carrera de Ingeniería en Sistemas computacionales).

Lazo, W. (2018). Diseño e implementación de un sitio web para el control de mantenimiento de equipos tecnológicos de la unidad de soporte y mantenimiento de la $\mathrm{ESPOCH}$ desarrollado en java con base de datos MYSQL en el período 2018.
López, L., Rosa, A., \& Quijije Anchundia, P. D. R. (2018). Análisis, desarrollo e implementación de una aplicación basada en tecnología web y móvil para la gestión de ventas y control de pedidos en línea en pastelería Dayana del cantón Montecristi (Doctoral dissertation).

Menéndez, M. (2015). Moon+ Reader. Disponible en: https://www.compartolid.es/m oon-reader/ Revisado el: 07/01/2015.

Pubgen. (2019). eBoox: lector de libros epub. Disponible en: http://pubgen.com/blog/eboox -reader-app-android/ Revisado el: 11/07/2017.

Santiago, R., \& Trabaldo, S. (2015). Mobile Learning: Nuevas realidades en el aula. DigitalText.

Travieso, J. L., \& Ribera, J. P. (2008). La alfabetización digital como factor de inclusión social: una mirada crítica. UOC Papers: revista sobre la sociedad del conocimiento, 6(7). 